\title{
LOS TRES PILARES FUNDAMENTALES EN LOS QUE SE APOYA EL MODELO ESPAÑOL DE PENSIONES: ¿HACIA DÓNDE VAMOS? ${ }^{1}$
}

\author{
Miguel Ángel Gómez Salado \\ Investigador Postdoctoral Contratado (acreditado como Profesor Contratado Doctor) \\ Departamento de Derecho del Trabajo y de la Seguridad Social \\ Universidad de Málaga
}

\begin{abstract}
En el presente estudio abordamos una cuestión relevante que, sin duda, se enmarca en el ámbito de la Seguridad Social: cómo está configurado en la actualidad nuestro modelo de pensiones en España. Para ello, efectuamos una primera aproximación al denominado Código de Lovaina, haciendo una especial mención a la vigente Constitución Española y a la propia Organización Internacional del Trabajo. Posteriormente, llevamos a cabo un análisis jurídico-social y económico referido a los tres pilares básicos en los que se apoya el sistema de pensiones de nuestro país. Por último, incorporamos algunas líneas a modo de conclusión.
\end{abstract}

In this study we address a relevant question that undoubtedly falls within the scope of Social Security: how our pension model is currently configured in Spain. To do this, we make a first approach to the so-called Leuven Code, making special mention of the current Spanish Constitution and the International Labor Organization itself. Subsequently, we carry out a legal-social and economic analysis referring to the three basic pillars on which the pension system of our country is based. Finally, we incorporate some lines to conclude.

IUSLabor 3/2021, ISSN 1699-2938, p. 98-124

DOI. 10.31009/IUSLabor.2021.i03.04

\footnotetext{
${ }^{1}$ El presente artículo se enmarca en las líneas de actuación de dos proyectos de investigación: Proyecto de I+D+i del Programa Estatal "Retos Investigación" orientado a los Retos de la Sociedad, "Retos, reformas y financiación del sistema de pensiones: ¿sostenibilidad versus suficiencia?” (ref. RTI2018-094696-B-I00), dirigido por Francisco VILA TIERNO y Miguel GuTIÉRREZ BENGOECHEA, y financiado por el Ministerio de Ciencia e Innovación; y Proyecto de $\mathrm{I}+\mathrm{D}+\mathrm{i}$ del Plan Andaluz de Investigación, Desarrollo e Innovación (PAIDI 2020) orientado a los Retos de la Sociedad Andaluza, "Los mayores en el contexto del empleo y la protección social: un reto para el crecimiento y el desarrollo económico" (ref. P18-RT-2585), dirigido por Francisco VILA TIERNo y Miguel GUTIÉRREZ BENGOECHEA, y cofinanciado por la Unión Europea (FEDER) y por la Junta de Andalucía. Del mismo modo, se ha elaborado a raíz de una estancia de investigación de carácter postdoctoral en la Universidad de Sevilla (2020).
} 
Title: The three fundamental pillars on which the Spanish pension model is based: where are we going?

Palabras clave: Derecho, España, pilares, previsión individual, protección social complementaria, previsión social empresarial, Seguridad Social, sistema de pensiones. Key words: Law, Spain, pillars, individual welfare, complementary social protection, business social welfare, Social Security, pension system.

Fecha envío: 24.8.9.2021 | Fecha aceptación: 7.10.2021

\section{Sumario}

1. Una aproximación inicial al denominado Código de Lovaina

2. Los tres pilares básicos del modelo español de pensiones: análisis jurídico-social y económico

2.1. Primer pilar: sistema público de previsión

2.2. Pilares segundo y tercero: sistema privado de previsión

2.2.1. Pilar privado-colectivo

2.2.2. Pilar privado-individual

3. Para concluir: ¿hacia dónde vamos?

4. Bibliografía 


\section{Una aproximación inicial al denominado Código de Lovaina}

En primer término, nos gustaría indicar que a partir de la década de 1960 comenzó a configurarse $^{2}$ un modelo conocido como teoría o esquema de los tres pilares (Código de Lovaina) en los que debe estar basado el sistema de pensiones de cualquier país: el primer pilar, evidentemente, hace mención al sistema público de previsión; el segundo pilar está conformado por los regímenes privados y complementarios de carácter ocupacional o de empleo, que se originan en el marco de las empresas y de las relaciones laborales; y el tercero, por su parte, está compuesto por las llamadas cuentas individuales de ahorro previsor a largo plazo.

Mediante los esfuerzos realizados por numerosos investigadores y organismos -tanto nacionales como internacionales-, se ha venido prestando una gran atención a este tema, poniéndose de manifiesto que la aplicación de la referida teoría puede conllevar una disminución sustancial de la vulnerabilidad de los sistemas de pensiones, así como un importante estímulo al ahorro por parte del ámbito familiar ${ }^{3}$.

Dicho esquema conceptual logra una significación desde la perspectiva jurídico-social y constitucional, y se percibe, una vez que se aplica en cada uno de los países, como una forma interesante de delimitar los diferentes sistemas de protección social. En la vigente Constitución Española ${ }^{4}$ (en lo sucesivo, CE), la teoría de los tres pilares fundamentales no aparece determinada ni descrita de manera expresa, pero sí que se encuentra presente de algún modo (aunque sea de manera difusa), concretamente en la redacción del artículo 41 de la $\mathrm{CE}$, donde se dispone que "los poderes públicos mantendrán un régimen público de Seguridad Social para todos los ciudadanos, que garantice la asistencia y prestaciones sociales suficientes ante situaciones de necesidad (...). La asistencia y prestaciones complementarias serán libres".

\footnotetext{
${ }^{2}$ DupeYroux, Jean Jacques, Évolution et tendances des systèmes de Sécurité Sociale des pays membres des Communautés Européennes et de la Grande-Bretagne, Services des publications des Communautés Européennes, Luxembourg, 1966 (disponible en: http://aei.pitt.edu/36107/1/A2119.pdf). Este autor ha sido uno de los primeros investigadores que enunciaron este concepto o esquema básico, que se conoce como Código de Lovaina. Sobre este tema, también se han pronunciado otros autores como: HERNÁNDEZ SOLís, Montserrat, Topa CANTISANO, Gabriela y HerRador AlCAide, Teresa Carmen, "Análisis de la actitud financiera personal para la jubilación: un sondeo en España”, Economía industrial, no 413, 2019, p. 105 y ss.; y SITJAR DE TOGORES CALVO, Ana Isabel, "Planes de pensiones: cuestiones transfronterizas. Una aproximación a la normativa y jurisprudencia de la Unión Europea", Cuadernos de información, vol. 7, 2009 , p. 115 y ss.

${ }^{3}$ Pieschacón Velasco, Camilo, Sistemas de pensiones: experiencia española e internacional, tomo II, Fundación Inverco, Madrid, 2005, p. 540.

${ }^{4}$ BOE $n^{\circ} 311$, de 29 de diciembre de 1978.
} 
Como se puede comprobar, este mandato a los poderes públicos se dirige a "mantener" un sistema público de Seguridad Social que se encargue, entre otras cosas, de garantizar unas pensiones que sean suficientes. Ahora bien, el denominado sistema público de la Seguridad Social no queda definido de manera "solipsista", ya que el mismo puede llegar a convivir con otros regímenes privados y complementarios de carácter libre, si bien estos últimos no pueden ser entendidos como equivalentes del primero. Así lo destaca la STC n $208 / 1988$, de 10 noviembre ${ }^{6}$, al disponer que "del artículo 41 de la CE (se) deriva una necesaria separación entre el régimen público de la Seguridad Social y las prestaciones complementarias «libres» basadas en una lógica contractual privada y, en consecuencia, financiables en principio con fondos privados y a cargo de los asegurados". Como ya se encargó de señalar el propio Tribunal Constitucional, la noción de Seguridad Social, teniendo en cuenta el marco normativo "interno e internacional, donde hoy se mueve la institución", "no puede predicarse de instituciones protectoras cuyo origen, tanto como la extensión de la acción tutelar que dispensan, descansa en la autonomía de la voluntad" .

En esta línea, cabe señalar que la Organización Internacional del Trabajo (en adelante, OIT) ha defendido también un sistema de pensiones más amplio, por ejemplo, cuando ha manifestado que "todos los países necesitan desarrollar diseños pluralistas y estructuras flexibles para sus sistemas de Seguridad Social. Para cumplir los objetivos de aliviar la pobreza en la vejez y proporcionar prestaciones de jubilación de bajo riesgo, generalmente se necesitan prestaciones que procedan de múltiples fuentes" ${ }^{\text {, o al decir }}$ que "para reducir el riesgo a través de la diversificación, el mejor enfoque para los países desarrollados puede ser caracterizado como un sistema multipilar, donde los pilares se determinen en base a su riesgo y a características redistributivas".

Asimismo, se puede destacar que la OIT ha venido promoviendo un sistema definido por cuatro pilares: un primer pilar para la lucha contra la pobreza, financiado con los ingresos

\footnotetext{
${ }^{5}$ MerCAdER Uguina, Jesús Rafael, “Artículo 41 CE: Régimen público de Seguridad Social para todos los ciudadanos”, Diario La Ley (disponible en: https://diariolaley.laleynext.es/d1l/2019/01/17/articulo-41-ceregimen-publico-de-seguridad-social-para-todos-los-ciudadanos).

${ }^{6}$ RTC $1988 \backslash 208$.

${ }^{7}$ Mercader Uguina, Jesús Rafael, “Artículo 41 CE: Régimen público de Seguridad Social para todos...”, $o b$ cit. Este autor también señala que, según la doctrina constitucional, el carácter necesariamente público del sistema de Seguridad Social no excluye el establecimiento de fórmulas de gestión o de responsabilidad privadas, siempre y cuando estas sean "de importancia relativa" en el conjunto de la gestión de aquel " $y$, consiguientemente, el papel y el compromiso de los poderes públicos en la satisfacción de su función protectora siga siendo "predominante»". Vid. SSTC $n^{\circ}$ 37/1994, de 10 de febrero (RTC 1994137), y 129/1994, de 5 de mayo (RTC 1994\129).

${ }^{8}$ Gillion, Colin, Turner, John, BaIley, Clive y LatulipPe, Denis, Social Security pensions: development and reform, International Labour Organization, Geneva, 2000, p. 18.

${ }^{9}$ Ibídem.
} 
generales del Estado y que se correspondería con las pensiones no contributivas o asistenciales; un segundo pilar contributivo, obligatorio y gestionado por el propio Estado; un tercer pilar que sería un componente de contribución definida y obligatorio hasta un cierto nivel, gestionado por las instituciones privadas; y un cuarto pilar de contribución definida y voluntario, también gestionado por las instituciones privadas ${ }^{10}$.

Teniendo en cuenta este esquema básico de los tres o cuatro pilares, los diversos países del mundo (entre ellos España) han logrado establecer distintas variantes para configurar sus propios modelos de pensiones. Así, con la finalidad de analizar nuestra experiencia nacional, seguidamente incluimos un apartado referido al sistema de pensiones en España ${ }^{11}$ donde podremos apreciar claramente cómo se ha aplicado este esquema teórico básico al modelo de pensiones de nuestro país.

\section{Los tres pilares básicos del modelo español de pensiones: análisis jurídico-social y económico}

En gran parte de los países de nuestro entorno, la dotación de recursos para hacer frente a algunas contingencias como la jubilación, la invalidez y la viudedad, entre otras, suele estar anclada en tres pilares o columnas. En función del país, la relevancia de cada uno de estos pilares podrá ser mayor o menor.

A continuación, exponemos cuáles son los tres principales pilares en los que se basa el sistema de pensiones en España. Sin duda alguna, podremos comprobar que en nuestro país, al igual que en otros países del sur de Europa, una gran parte de las pensiones son públicas, si bien también encontramos diversos instrumentos de previsión social complementaria que están adquiriendo un rápido e importante desarrollo.

\subsection{Primer pilar: sistema público de previsión}

El primer pilar se encuentra regulado en el Real Decreto Legislativo 8/2015, de 30 de octubre, por el que se aprueba el texto refundido de la Ley General de la Seguridad Social $^{12}$ y en su normativa de desarrollo.

\footnotetext{
${ }^{10}$ Ibídem.

${ }^{11}$ Un trabajo reciente que trata sobre la situación actual de nuestro sistema de pensiones, haciendo hincapié en el envejecimiento poblacional, es el elaborado por AGUILAR SEGADO, Carlos David, "Situación actual de las pensiones en España: perspectiva económica-financiera", Revista de Estudios Jurídico Laborales y de Seguridad Social, $\mathrm{n}^{\circ}$ 2, 2021, p. 279-295.

${ }^{12} \mathrm{BOE} \mathrm{n}^{\circ} 261$, de 31 de octubre de 2015.
} 
Este pilar está representado por la propia Seguridad Social, mediante la cual el Estado permite cubrir un conjunto de contingencias relacionadas con la vejez (jubilación), el fallecimiento (viudedad, orfandad y en favor de familiares) y la enfermedad (incapacidad).

Sin duda alguna, de todos los riesgos cubiertos por la Seguridad Social consistentes en defectos de rentas, el más relevante es el de la vejez. Y ello, porque se trata de una contingencia que se caracteriza "por la frecuencia de su cristalización, al constituir el término previsible y normal de la vida profesional del individuo, tan sólo alterada por situaciones cuantitativamente irrelevantes de invalidez y muerte. Podemos decir, por tanto, que la jubilación es receptora, en términos generales, del conjunto de ciudadanos que se incorporan a la población activa, de ahi la importante dependencia de las estructuras demográficas de cada país y de los fenómenos que inciden sobre aquéllas" ${ }^{13}$.

Por otro lado, debemos señalar que este primer pilar, es decir, el modelo público de pensiones de la Seguridad Social continúa siendo en la actualidad el elemento principal del sistema español de previsión social y presenta, entre otros, los siguientes caracteres ${ }^{14}$.

a. Tiene un carácter obligatorio.

b. Se configura como un sistema mayoritariamente contributivo con una serie de elementos asistenciales. Ello conlleva, evidentemente, la existencia de una relación teórica de proporcionalidad entre lo que se tiene derecho a percibir y lo que se ha cotizado o contribuido, de tal manera que aquellas personas que no han contribuido (o no lo han hecho de manera suficiente) con sus aportaciones solo tendrán derecho a los servicios sanitarios/sociales y a una serie de prestaciones no contributivas.

c. Está asentado fundamentalmente en un régimen público (financiado mediante reparto $^{15}$ ), basado en el principio de solidaridad intergeneracional. Esto supone que

13 TORTUERo PlazA, José Luis, "Los retos históricos del sistema de pensiones proyectados en tiempos de crisis económico financiera", Areas: revista internacional de ciencias sociales, no 32, 2013, p. 56.

14 En este mismo sentido, vid. los siguientes trabajos, entre otros: MonEREO PÉrEZ, José Luis, OJEDA AVILÉs, Antonio y GuTIÉRREZ BENGOECHEA, Miguel, Reforma de las pensiones públicas y planes privados de pensiones, Laborum, Murcia, 2021, p. 7-39; InSTITUTO BBVA DE PENSIONES, "Los tres pilares de la previsión social: del ámbito público al privado", 2019 (disponible en: https://www.jubilaciondefuturo.es/es/blog/los-tres-pilares-de-la-prevision-social-del-ambito-publico-alprivado.html); REDACCIÓN DE EKONOMIAZ, "Guía de la previsión social complementaria”, Revista vasca de economía (Ekonomiaz), no 85, 2014, p. 287-288; y MONEREO PÉREZ, José Luis et al., Manual de Seguridad Social, Tecnos, Madrid, 2020.

${ }^{15}$ Este sistema exige, según Francisco VILA y Miguel GuTIÉRREZ, "una fuerte vinculación del pensionista con el trabajador a través de un contrato social entre generaciones, por lo que es esencial un equilibrio 
el dinero que se recauda cada año con las cotizaciones de los empleados activos (y de los empleadores que los han tenido contratados) y otros mecanismos impositivos, sirve para el pago de las prestaciones públicas que se satisfacen ese mismo año a los beneficiarios (pasivos o pensionistas) ${ }^{16}$. Asimismo, las cotizaciones sociales de los empresarios y trabajadores activos se utilizan para mantener el llamado Fondo de Reserva $^{17}$ (pura capitalización), habitualmente conocido como "hucha" de las pensiones, cuyo objetivo es conformar un colchón financiero que permita cubrir las necesidades futuras en materia de prestaciones contributivas ocasionadas por desviaciones entre ingresos y gastos de la Seguridad Social (como se sabe, el envejecimiento de la población, unido a la grave crisis económica que ha sufrido España en los últimos años y al dato objetivo del descenso de las cotizaciones sociales, ha motivado ${ }^{18}$ que el Gobierno haya tenido ya que recurrir en numerosas ocasiones a este fondo)

d. Está gestionado por el Estado.

entre población ocupada y jubilada que está igualmente ligado a la relación existente entre natalidad y mortalidad". Vid. VILA TIERNO, Francisco, "El difícil equilibrio entre la sostenibilidad y la suficiencia. Una visión general dela situación general del sistema de pensiones pensando en el futuro", en AA.VV. (Vila Tierno, Francisco, y Gutiérrez Bengoechea, Miguel, (Directores); Gómez Salado, Miguel Ángel, Coordinador), La incidencia de los diferentes factores endógenos y exógenos sobre sostenibilidad y suficiencia en el sistema de pensiones, Comares, Granada, 2020, p. 12; VILA TIERNO, Francisco y MORENO ROMERO, Francisca, "Equilibrio entre suficiencia y sostenibilidad: pensiones mínimas, revalorización automática y factor de sostenibilidad", en AA.VV. (ÁlvAREZ CoRTÉs, Juan Carlos, Director), Trabajadores maduros y Seguridad Social, Thomson Reuters Aranzadi, Cizur Menor, 2018, p. 69-92; y GuTIÉRREZ BENGOECHEA, Miguel, "El sistema de reparto de la Seguridad Social versus envejecimiento poblacional", Revista de Estudios Jurídico Laborales y de Seguridad Social, n ${ }^{\circ}$, 2020, p. 235 y ss.

${ }^{16}$ Por tanto, en un sistema de reparto como el de España, las pensiones públicas actuales son pagadas con las cotizaciones y los impuestos de aquellas personas -especialmente las más jóvenes- que trabajan en la actualidad, mientas que las pensiones futuras se pagarán con el dinero que se recaude con los trabajadores de ese momento. En esta línea, señala David NATALI que el método de financiación de nuestro sistema español es el modelo "de reparto", según el cual "las cotizaciones actuales que los trabajadores y los empresarios pagan (o los ingresos procedentes de la recaudación de impuestos actual) no se acumulan, sino que se utilizan inmediatamente para financiar las prestaciones actuales". NATALI, David, "La reforma de las pensiones en la Unión Europea: Cambios en las políticas nacionales y coordinación de la Unión Europea", en AA.VV. (Frades Pernas, Jaime, Coordinador), El sistema público de pensiones de jubilación. Desafios y respuestas, Fundación Largo Caballero, Madrid, 2011, p. 479.

${ }^{17}$ Creado en el año 2000 en el marco del Pacto de Toledo.

${ }^{18}$ SÁNCHEZ-RODAS NAVARRO, Cristina, "La cuadratura del círculo: sostenibilidad del sistema de pensiones y desempleo juvenil”, Revista Galega de Dereito Social, nº 2, 2016, p. 67. 
Una vez expuesto lo anterior, parece conveniente señalar que el actual sistema público de pensiones español (vid. gráfica 1) está conformado por dos subsistemas principales ${ }^{19}$ :

\section{Gráfica 1: Primer pilar (Seguridad Social)}

Público y obligatorio

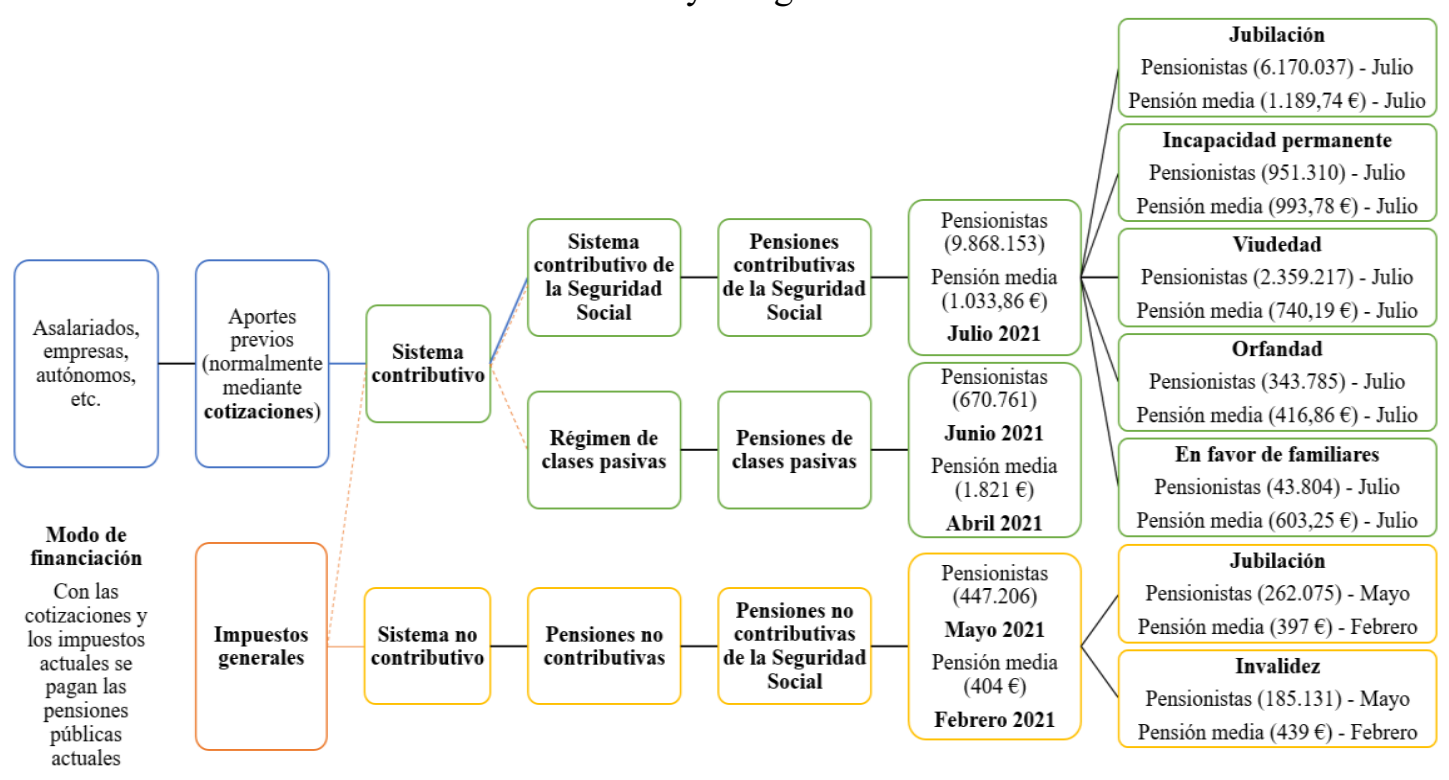

Gráfica de elaboración propia ${ }^{20}$.

a. Un sistema contributivo: reconoce pensiones que tienen la finalidad de garantizar la protección ante determinadas contingencias (como la enfermedad y el fallecimiento) $\mathrm{y}$ ante determinadas situaciones vitales, como la jubilación. Estas proveen un determinado nivel de prestaciones (en virtud de ello, se conocen como pensiones de

\footnotetext{
${ }^{19}$ Ahora bien, hemos encontrado otros trabajos en los que se establece esta otra clasificación (también aceptada por la doctrina científica): a. sistema contributivo; b. sistema no contributivo; y c. sistema de clases pasivas.

${ }^{20}$ Para la elaboración de esta imagen, hemos partido de los datos obtenidos en los siguientes portales web: a) sistema contributivo: Gobierno de España, "La nómina de las pensiones contributivas a 1 de julio se sitúa en 10.202,29 millones de euros", 27 de julio de 2021 (disponible en: https://www.lamoncloa.gob.es/serviciosdeprensa/notasprensa/inclusion/Paginas/2021/270721pensiones_contributivas.aspx); y Gobierno de España, "Estadísticas e informes (clases pasivas)" (disponible en: https://www.portalclasespasivas.gob.es/sitios/clasespasivas/esES/QuienesSomos/EstadisticasInformes/Paginas/EstadisticasInformes.aspx). b) sistema no contributivo: Gobierno de España, "Estadísticas (Prestaciones no contributivas)", Boletín de Estadísticas Laborales (disponible en: https://www.mites.gob.es/es/estadisticas/prestaciones_SS_otra_proteccion/PNC/welcome.htm); y La Información, "La prestación media es de 404 euros: El 35\% de las pensiones del Imserso se queda en un cajón... Estas son las causas", 2021 (disponible en: https://www.lainformacion.com/economia-negocios-yfinanzas/solicitar-pension-contributiva-invalidez-jubilacion-dinero-imserso/2833332/).
} 
"prestación definida" ${ }^{21}$ ) a todas aquellas personas que han contribuido al sistema con aportes previos -normalmente mediante cotizaciones- durante un número de años determinado (o a sus beneficiarios, en el supuesto de fallecimiento) ${ }^{22}$.

El sistema contributivo tiene, a su vez, estos dos niveles ${ }^{23}$ :

a.1. Un sistema contributivo de la Seguridad Social: este primer sistema, diseñado originalmente en la década de 1960, posibilita el reconocimiento de prestaciones económicas y de duración indefinida, aunque no siempre, por vejez (jubilación), enfermedad (incapacidad), muerte y supervivencia (viudedad, orfandad y en favor de familiares), cuya concesión está por lo general supeditada a una previa relación jurídica con la Seguridad Social (acreditar un período mínimo de cotización en determinados casos), siempre que se cumplan los demás requisitos requeridos ${ }^{24}$.

\footnotetext{
${ }^{21}$ Los sistemas de pensiones de "prestación definida" (que no de "aportación definida"), si bien adoptan distintas formas, básicamente consisten en que los trabajadores financian con sus aportes previos normalmente mediante cotizaciones- "las pensiones de los jubilados en ese momento, y la pensión que reciben se establece según unos parámetros predeterminados y conocidos, por lo que la prestación está definida con antelación. Por ejemplo, en función de los años cotizados y el último salario o en función del mejor salario entre un periodo de años cotizados. El sistema de pensiones español, por poner un ejemplo, es un sistema de prestación definida". Vid. RodRÍGUEZ CANFRANC, Miguel, "¿Qué son los sistemas de pensiones de aportación definida?", $B B V A$, agosto de 2020 (disponible en: https://www.bbva.com/es/queson-los-sistemas-de-pensiones-de-aportacion-definida/).

${ }^{22}$ Dentro del sistema contributivo, podemos encontrar un régimen general, así como varios regímenes especiales.

${ }^{23}$ Hemos comprobado que la clasificación del sistema contributivo en estos dos niveles diferenciados también ha sido incluida en otros trabajos como el de RodríGUEz CABRERO, Gregorio, "La reforma del sistema público de pensiones en España", Documentos de trabajo (CSIC. Unidad de Políticas Comparadas), no 13, 2002, p. 3. Vid. también, RODRÍGUEZ CABRERO, Gregorio, "La reforma permanente del sistema público de pensiones", en AA.VV., Actores sociales y reformas del bienestar, CSIC, Madrid, 2005, p. 27-52.

${ }^{24}$ También se hace mención a los requisitos requeridos para el acceso a las pensiones contributivas en España en los siguientes trabajos, entre otros: MORENO ROMERO, Francisca, "Requisitos generales de acceso a la protección y sus vicisitudes: hacia un necesario replanteamiento", en AA.VV. (VILA TIERNO, Francisco, y GutiérRez Bengoechea, Miguel, (Directores); Gómez Salado, Miguel Ángel, Coordinador), La incidencia de los diferentes factores endógenos y exógenos sobre sostenibilidad y suficiencia en el sistema de pensiones, Comares, Granada, 2020, p. 115-132; y RUIZ SANTAMARÍA, José Luis, "Las pensiones en el marco del actual Convenio Bilateral de Seguridad Social suscrito entre España y Colombia", e-Revista internacional de la protección social, vol. 3, nº 2, p. 244.
} 
Este sistema, cuyas pensiones contributivas están financiadas fundamentalmente con las cotizaciones o cuotas abonadas por los trabajadores y empresarios ${ }^{25}$, es el más importante y el que siempre ocupa el centro del debate político nacional, ya que su número de pensionistas se acerca progresivamente a los 10.000.000 de personas en España (concretamente, en el mes de julio de 2021, el número de pensionistas ascendió a, nada más y nada menos, que 9.868 .153 personas; vid. gráfica 1).

Se puede destacar que la pensión media del sistema contributivo de la Seguridad Social (vid. gráfica 2) se situó en 1.033,86 € en julio de 2021. Esta cifra, que abarca los diferentes tipos de pensión (jubilación, incapacidad permanente, viudedad, orfandad y en favor de familiares), se vio incrementada en el último año en un $2,18 \%$. La pensión media (contributiva de la Seguridad Social) de los hombres fue de 1.254,71 $€$ en dicho mes, mientras que la mensualidad percibida por las mujeres ascendió de media a $831,44 €$.

Gráfica 2: Evolución del importe de la pensión media en España Mes de julio. Total del sistema contributivo de la Seguridad Social

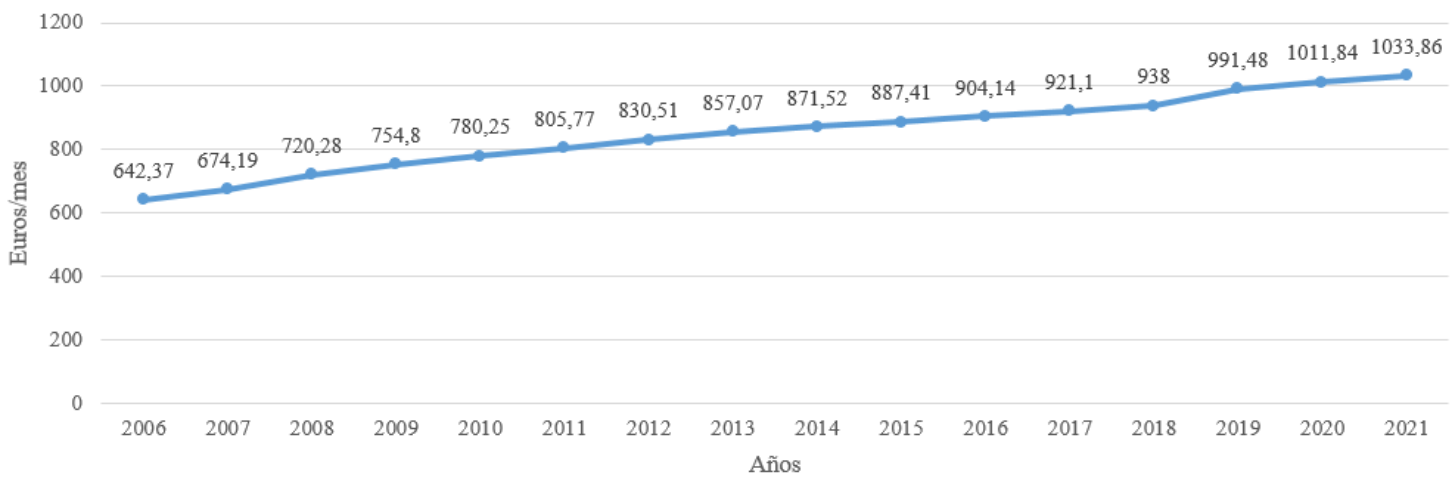

Gráfica de elaboración propia ${ }^{26}$.

En particular, podemos decir que la pensión media de jubilación del sistema fue de $1.189,74 €$ en julio de 2021 (vid. gráfica 1), lo que evidencia un aumento del $2,30 \%$ en relación al del mismo mes de 2020 . Por su parte, la pensión media de viudedad fue de 740,19 euros al mes (vid. gráfica 1).

\footnotetext{
${ }^{25}$ Estas se obtienen a través de la aplicación de determinados porcentajes o tipos sobre una base de cotización calculada sobre el salario percibido o, en su caso, tarifada o fija, en función del riesgo o contingencia objeto de protección.

${ }^{26}$ La gráfica se ha elaborado a partir de los datos incluidos en la siguiente nota de prensa del Ministerio de Inclusión, Seguridad Social y Migraciones: Gobierno de España, "La nómina de las pensiones contributivas a 1 de julio se sitúa en 10.202,29 millones de euros", 27 de julio de 2021 (disponible en: https://www.lamoncloa.gob.es/serviciosdeprensa/notasprensa/inclusion/Paginas/2021/270721pensiones_contributivas.aspx).
} 
En definitiva, el sistema contributivo de la Seguridad Social, cuyas pensiones se encuentran financiadas principalmente con las cotizaciones o cuotas que abonan los trabajadores y empresarios, ocupa una posición dominante en el modelo de pensiones español. Esta posición destacada se ve reflejada particularmente en el elevado número de pensiones que ofrece mensualmente (cerca de 10 millones de pensiones contributivas abonadas, como ya se ha indicado ${ }^{27}$ ) y en la importante nómina mensual de las prestaciones contributivas de la Seguridad Social (más de 10.200 millones de euros ${ }^{28}$; vid. tabla 1$)$.

Tabla 1: Importe mensual de la nómina de pensiones contributivas de la Seguridad Social en España (en miles de euros)

Pensiones en vigor a día 1 de cada mes

\begin{tabular}{|c|c|c|c|c|c|c|c|}
\hline \multicolumn{2}{|c|}{ Periodo } & $\begin{array}{l}\text { Incapacidad } \\
\text { permanente }\end{array}$ & Jubilación & Viudedad & Orfandad & F. Familiar & Total \\
\hline \multirow{12}{*}{2020} & Ene & 939.764 & 6.975 .564 & 1.690 .756 & 137.868 & 25.039 & 9.768 .990 \\
\hline & Feb & 945.690 & 7.056 .005 & 1.706 .215 & 139.178 & 25.233 & 9.872 .321 \\
\hline & Mar & 945.839 & 7.060 .520 & 1.706 .549 & 139.552 & 25.315 & 9.877 .775 \\
\hline & Abr & 943.806 & 7.064 .534 & 1.705 .849 & 139.617 & 25.355 & 9.879 .161 \\
\hline & May & 940.178 & 7.049 .446 & 1.698 .649 & 139.195 & 25.312 & 9.852 .781 \\
\hline & Jun & 937.750 & 7.057 .662 & 1.702 .316 & 139.293 & 25.329 & 9.862 .349 \\
\hline & Jul & 936.927 & 7.072 .760 & 1.708 .029 & 139.535 & 25.410 & 9.882 .662 \\
\hline & Ago & 936.228 & 7.092 .191 & 1.710 .389 & 139.801 & 25.419 & 9.904 .029 \\
\hline & Sep & 934.109 & 7.103 .243 & 1.708 .997 & 139.620 & 25.456 & 9.911 .425 \\
\hline & Oct & 933.248 & 7.121 .518 & 1.710 .741 & 139.137 & 25.469 & 9.930 .113 \\
\hline & Nov & 932.897 & 7.144 .386 & 1.713 .309 & 138.979 & 25.520 & 9.955 .091 \\
\hline & Dic & 934.831 & 7.168 .760 & 1.716 .601 & 139.481 & 25.586 & 9.985 .260 \\
\hline \multirow{7}{*}{2021} & Ene & 943.238 & 7.246 .794 & 1.731 .033 & 140.771 & 25.861 & 10.087 .697 \\
\hline & Feb & 941.036 & 7.262 .417 & 1.730 .238 & 140.992 & 25.837 & 10.100 .521 \\
\hline & Mar & 941.425 & 7.277 .049 & 1.733 .762 & 141.410 & 25.942 & 10.119 .588 \\
\hline & Abr & 941.360 & 7.289 .055 & 1.737 .843 & 141.906 & 26.032 & 10.136 .196 \\
\hline & May & 942.060 & 7.303 .066 & 1.740 .518 & 142.375 & 26.118 & 10.154 .137 \\
\hline & Jun & 944.093 & 7.322 .908 & 1.744 .071 & 142.884 & 26.273 & 10.180 .229 \\
\hline & Jul & 945.580 & 7.340 .712 & 1.746 .269 & 143.309 & 26.425 & 10.202 .294 \\
\hline
\end{tabular}

Tabla de elaboración propia ${ }^{29}$.

a.2. Un sistema de clases pasivas del Estado: este sistema, con casi 200 años de antigüedad $^{30}$, forma parte del llamado Régimen Especial de la Seguridad Social

${ }^{27}$ Como ya hemos señalado, a 1 de julio de 2021 fueron abonadas 9.868 .153 pensiones contributivas (un $1,04 \%$ más que en julio de 2020).

${ }^{28}$ La nómina mensual de las prestaciones contributivas de la Seguridad Social se ha situado en 10.202,29 millones de euros a 1 de julio, lo que supone un 3,23\% más que en el mismo mes del año anterior. Más de dos tercios de la nómina, 7.340,71 millones de euros, fueron destinados al pago de las pensiones de jubilación. Esta cuantía experimentó un crecimiento del 3,79\% en los últimos doce meses.

${ }^{29}$ La tabla se ha elaborado a partir de los datos incluidos en la siguiente nota de prensa del Ministerio de Inclusión, Seguridad Social y Migraciones: Gobierno de España, "La nómina de las pensiones contributivas a 1 de julio se sitúa en 10.202,29 millones de euros”, 27 de julio de 2021 (disponible en: https://www.lamoncloa.gob.es/serviciosdeprensa/notasprensa/inclusion/Paginas/2021/270721 pensiones_contributivas.aspx).

${ }^{30}$ En algunos trabajos se indica que el nacimiento del sistema de clases pasivas se sitúa concretamente en el año 1835. Vid. BERRAQUERO ESCRIBANO, Iván, "La supresión de las clases pasivas. Implicaciones a largo plazo para las Comunidades Autónomas”, ASOCEX (disponible en: https://asocex.es/la-supresion-de-lasclases-pasivas-implicaciones-a-largo-plazo-para-las-comunidades-autonomas). 
de los Funcionarios y garantiza la protección frente a los riesgos de vejez, incapacidad, muerte y supervivencia de determinados colectivos ${ }^{31}$ que prestan o han prestado servicios al Estado. Desde el día 1 de enero de 2011, el personal de nuevo ingreso en los cuerpos y escalas mencionados en la nota a pie de página inmediatamente anterior se integra en el Régimen General de la Seguridad Social $^{32}$ (esto significa que este sistema continúa en vigor pero, desde 2011, no incorpora nuevos funcionarios, lo que provocará su extinción futura).

La nómina mensual de pensiones de clases pasivas ascendió a 2.421,08 millones de euros en el mes de junio de 2021. El incremento sobre el mes anterior fue de $98,45 \%$, ya que incorpora la extra de junio. La variación interanual fue de $5,23 \%{ }^{33}$.

En la nómina del referido mes de junio, el número de pensionistas del régimen de clases pasivas ascendió a 670.761 personas (su importe medio se situó en abril del mismo año en $1.821 €$; vid. gráfica 1). En la actualidad, estas pensiones se financian con ingresos procedentes de los impuestos generales ${ }^{34}$.

b. Un sistema no contributivo o asistencial: se creó en 1990 y reconoce prestaciones, fundamentalmente de jubilación e invalidez (que se financian mediante los impuestos generales, con cargo a las partidas correspondientes de los Presupuestos Generales del Estado), a aquellas personas que, encontrándose en una situación de necesidad protegible, carezcan de los recursos suficientes para su subsistencia en los términos legalmente fijados, aun cuando no hayan cotizado nunca o el tiempo suficiente para alcanzar las prestaciones del nivel contributivo.

\footnotetext{
${ }^{31}$ Están incluidos: funcionarios de carrera de la Administración General del Estado, de la Administración de Justicia, de las Cortes Generales, de otros órganos constitucionales o estatales que lo prevean y funcionarios transferidos a las Comunidades Autónomas (que no ingresen en cuerpos propios de las mismas); militares de carrera, de las escalas de complemento, de tropa y marinería profesional y los caballeros cadetes, alumnos y aspirantes de las escuelas y academias militares; expresidentes, vicepresidentes y exministros del Gobierno de la nación y otros altos cargos.

${ }^{32}$ Con esta medida, el legislador ha pretendido incrementar el número de cotizantes a la Seguridad Social $\mathrm{y}$, asimismo, extinguir progresivamente el sistema de clases pasivas (en lo que se refiere al subsistema de pensiones).

${ }^{33}$ Gobierno de España, "La nómina de las pensiones contributivas a 1 de julio se sitúa en 10.202,29 millones de euros", 27 de julio de 2021 (disponible en: https://www.lamoncloa.gob.es/serviciosdeprensa/notasprensa/inclusion/Paginas/2021/270721 pensiones_contributivas.aspx).

${ }^{34}$ Los fondos para abonar las prestaciones correspondientes de las clases pasivas proceden de los Presupuestos Generales del Estado.
} 
Las pensiones no contributivas ${ }^{35}$ de la Seguridad Social solo llegaron a 447.206 en mayo de 2021 y su importe medio se situó en aproximadamente $404 €$ en el mes de febrero del mismo año (a diferencia de las pensiones contributivas de la Seguridad Social, cuyo importe medio alcanzó, como hemos visto, los 1.033,86€ en julio de 2021; vid. gráficas 1 y 2).

Aunque estas pensiones están bajo el control de la Seguridad Social, se gestionan por los órganos competentes de las Comunidades Autónomas y las Direcciones provinciales del Instituto de Mayores y Servicios Sociales (IMSERSO) en las ciudades de Ceuta y Melilla.

\subsection{Pilares segundo y tercero: sistema privado de previsión}

Dentro del sistema español de pensiones, tal y como se refleja en la CE, es posible distinguir dos bloques muy relevantes, las pensiones públicas obligatorias (estudiadas anteriormente), ya sean contributivas o no contributivas, y las pensiones privadas complementarias y voluntarias. Estas segundas, las pensiones privadas, configuran la llamada previsión o protección social complementaria, que conforma los denominados segundo y tercer pilares del sistema, cuya regulación se encuentra recogida básicamente en el Real Decreto Legislativo 1/2002, de 29 de noviembre, por el que se aprueba el texto refundido de la Ley de Regulación de los Planes y Fondos de Pensiones ${ }^{36}$, así como en su normativa de desarrollo.

Cabe señalar que la cobertura de estos otros dos pilares, es decir, de la previsión social privada, se realiza a través de las aportaciones a diferentes instrumentos (como los planes de pensiones ${ }^{37}$, los seguros, las mutualidades... que luego estudiaremos), en régimen de capitalización.

\footnotetext{
${ }^{35}$ Para profundizar sobre este tipo de pensiones, vid. los trabajos de MALDONADO MolinA, Juan Antonio, "Las pensiones no contributivas", en AA.VV., Tratado de Derecho de la Seguridad Social, tomo II, Laborum, Murcia, 2017, p. 285-304; y Álvarez CoRTÉs, Juan Carlos, "La pensión de jubilación no contributiva: su futuro papel de malla de seguridad social ante el repliegue contributivo", en AA.VV. (Álvarez Cortés, Juan Carlos, Director), Trabajadores maduros y Seguridad Social, Thomson Reuters Aranzadi, Cizur Menor, 2018, p. 265-299.

${ }^{36} \mathrm{BOE} \mathrm{n}^{\circ} 298$, de 13 de diciembre de 2002.

${ }^{37}$ Para quienes deseen profundizar en el tema de los planes de pensiones, nos permitimos recomendar los siguientes trabajos: Monereo PÉREZ, José Luis y FernÁndeZ BERnAT, Juan Antonio, "Planes y fondos de pensiones", en AA.VV., Tratado de Derecho de la Seguridad Social, tomo II, Laborum, Murcia, 2017, p. 807-824; Moreno GenÉ, Josep y Romero Burillo, Ana María, "Los planes y fondos de pensiones ante las nuevas formas de organización de la empresa", Revista de trabajo y seguridad social CEF, $\mathrm{n}^{\circ}$ 245-246, 2003, p. 15-74; y GUTIÉRREZ BEnGOeCHEA, Miguel, "Fiscalidad de los planes de pensiones privados", Revista de Derecho de la Seguridad Social, nº 26, 2021, p. 255-279.
} 
A pesar de que la protección social complementaria está presente en nuestro país desde hace muchos años, no es hasta el año 1987 (época en la cual "la situación económicofinanciera de la Seguridad Social era bastante crítica") ${ }^{38}$, en el momento en el que fue aprobada la Ley 8/1987, de 8 de junio, de Regulación de los Planes y Fondos de Pensiones ${ }^{39} 40$, cuando realmente se hace un intento de promover los diversos sistemas de previsión complementarios, para favorecer su extensión y desarrollo.

Dicho lo anterior, podemos señalar que los caracteres más importantes de la protección social complementaria en España son los siguientes ${ }^{41}$ :

a. Tiene un carácter voluntario y libre.

b. Genera un capital no sustitutivo de las pensiones públicas a las que se puede tener derecho en el régimen correspondiente de la Seguridad Social, teniendo, en consecuencia, un carácter privado y complementario de aquellas.

c. Está basada en un régimen privado (financiado mediante capitalización), frente al régimen público de reparto (financiado mediante reparto). Esto conlleva la realización de aportaciones previas durante la vida activa y el pago de la prestación en el momento en que sobrevenga la contingencia protegida, de tal manera que la cuantía de la pensión se corresponde con los recursos acumulados (es decir, las cuotas que han ido abonando las personas y entidades asociadas) más los intereses o rendimientos de dichos capitales (que participan de las fluctuaciones del mercado). O lo que es lo mismo, la pensión se concibe en este caso como una renta que procede del ahorro acumulado.

d. Está gestionada por entidades privadas.

Una vez expuesto lo anterior, pasamos a realizar un análisis de los pilares segundo y tercero.

\footnotetext{
${ }^{38}$ Según García PAdrón, Yaiza y García BozA, Juan, "Análisis legislativo de los planes y fondos de pensiones en España. Instauración y desarrollo", Ciencia y Sociedad, vol. XXXI, n 2, 2006, p. 259.

${ }^{39} \mathrm{BOE} \mathrm{n}^{\circ} 137$, de 9 de junio de 1987.

${ }^{40}$ Ahora bien, tal y como señalan algunos autores, esta norma "no estuvo operativa hasta 1988, cuando se publicó su Reglamento de desarrollo". Vid. PiesChaCón Velasco, Camilo, La Ley de Planes y Fondos de Pensiones: 20 Años después, Fundación Inverco, Madrid, 2007, p. VII.

${ }^{41}$ En este sentido, vid. los siguientes trabajos, entre otros: MonEREo PÉreZ, José Luis, OJEDA AVILÉs, Antonio y GutiérRez BengoecheA, Miguel, Reforma de las pensiones públicas..., ob cit., p. 7 y ss.; InStituto BBVA de Pensiones, "Los tres pilares de...", 2019, ob cit.; RedACCiÓn de EKonOMiAz, "Guía de la previsión social...”, ob cit., p. 289 y ss.; y MONEREO PÉREZ, José Luis et al., Manual de Seguridad..., ob cit.
} 


\subsubsection{Pilar privado-colectivo}

Dentro de la previsión social complementaria, se encuentra el segundo pilar ${ }^{42}$ (vid. gráfica 3), referido a los sistemas profesionales de protección social complementaria de carácter colectivo $^{43}$. Dicho en otras palabras, ello significa que el segundo pilar está conformado por los sistemas de pensiones promovidos por las empresas o instituciones con sus empleados y que están dirigidos a generar un capital complementario a las futuras pensiones públicas.

\section{Gráfica 3: Segundo pilar (Previsión social empresarial)}

Privado, colectivo y voluntario

Principales magnitudes (2019)

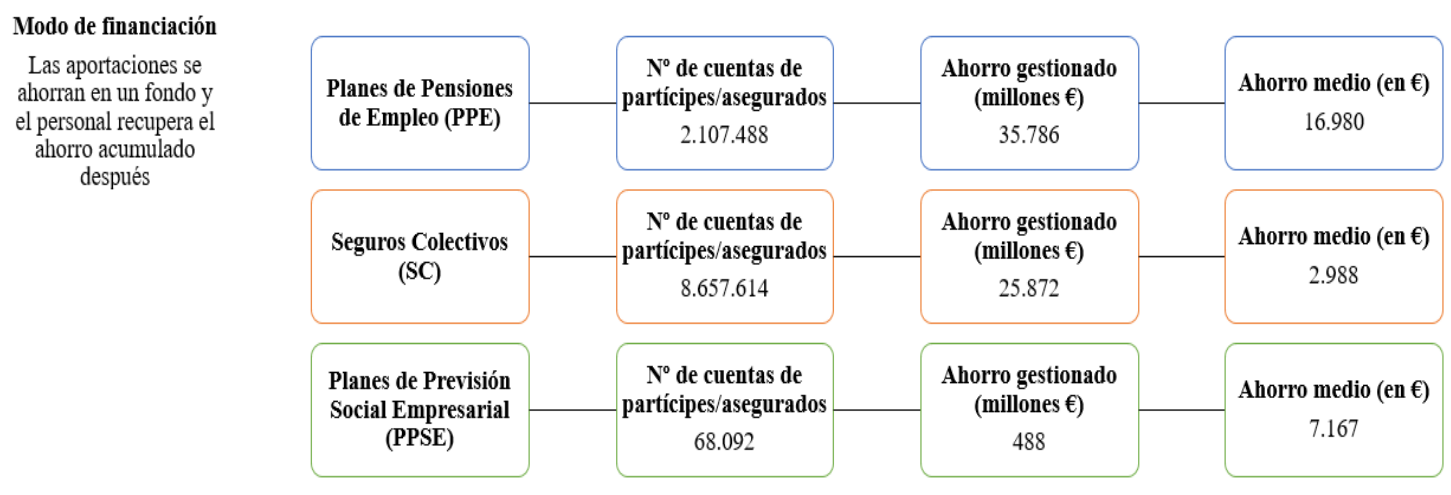

Gráfica de elaboración propia ${ }^{44}$.

Este segundo pilar, en contraste con el primero (público, obligatorio y basado en el régimen de reparto), se caracteriza por ser privado, voluntario y estar asentado en el sistema de capitalización, según el cual las contribuciones y aportaciones que realizan las empresas (o instituciones) y los empleados a lo largo del tiempo sirven para financiar las futuras pensiones y los resultados logrados de su inversión ${ }^{45}$. De igual modo, podemos señalar que no está presente en este sistema el elemento de solidaridad intergeneracional del primer pilar.

Por otro lado, cabe destacar que la financiación de este sistema de previsión social complementaria de carácter empresarial, cuyos beneficiarios son los empleados, suele ser

\footnotetext{
${ }^{42}$ El ahorro complementario a través de la compañía.

${ }^{43}$ Monereo PÉREZ, José Luis, "La garantía de las pensiones: desafíos para la sostenibilidad económica y social”, Revista de Estudios Jurídico Laborales y de Seguridad Social, n 3, 2021.

${ }^{44}$ Para la elaboración de esta gráfica, hemos partido de los datos contenidos en el Informe Estadístico de Instrumentos de Previsión Social Complementaria (2019 y avance 2020), publicado por el propio Ministerio de Asuntos Económicos y Transformación Digital.

${ }^{45}$ Las futuras prestaciones, por tanto, no van a depender únicamente de la cuantía de las aportaciones realizadas, sino también de la evolución financiera y temporal de dichas aportaciones.
} 
asumida por el promotor (el empleador), estando también admitida la contribución de los partícipes (los trabajadores).

En la actualidad, la cobertura de este pilar privado-colectivo se realiza mediante aportaciones (de las entidades, como hemos indicado, y en favor de los empleados) a diversos instrumentos que se definen a continuación: planes de pensiones de empleo, seguros colectivos que exteriorizan compromisos por pensiones, planes de previsión social empresarial y mutualidades de previsión social empresarial. A finales de 2019, estos instrumentos (vid. gráfica 4) tenían un patrimonio bajo gestión de 62.146 millones de euros y un número acumulado de cuentas de partícipes/asegurados de 10 millones ochocientos treinta y tres mil ciento noventa y cuatro cuentas.

\section{Gráfica 4: Ahorro gestionado}

Peso que en el año 2019 tenían los distintos instrumentos en el ahorro gestionado mediante el

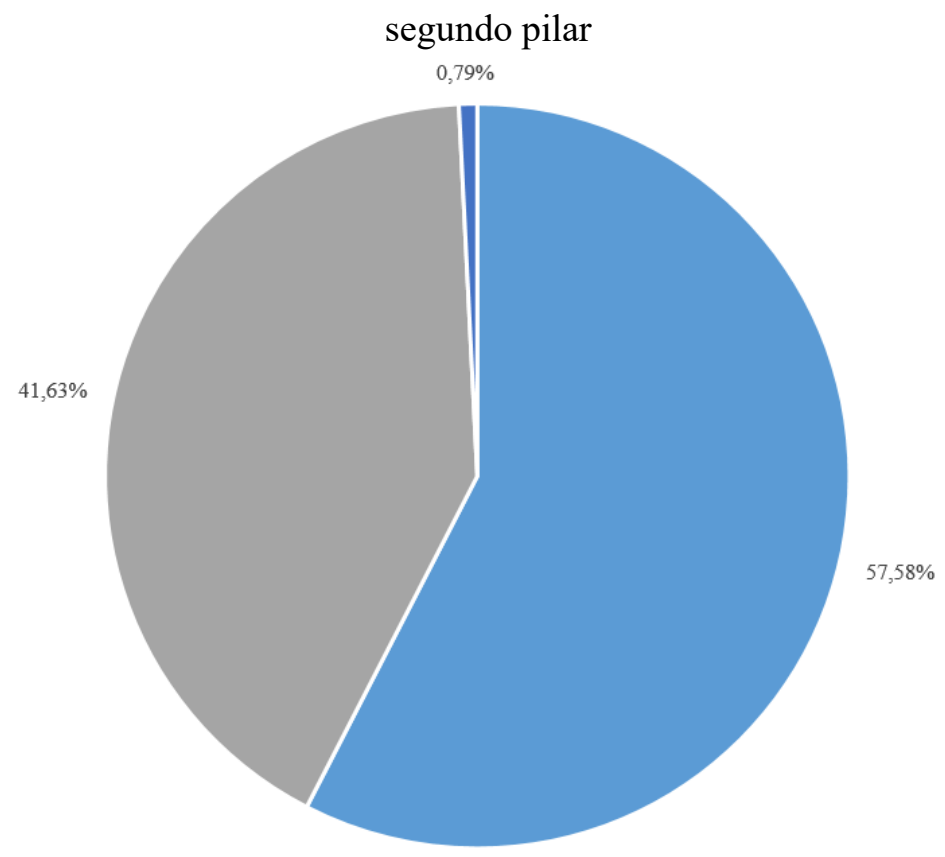

- PPE $-\mathrm{SC}$ - PPSE

Gráfica de elaboración propia ${ }^{46}$.

a. Planes de pensiones de empleo (PPE): están presentes en nuestro país desde la promulgación de la Ley de Planes y Fondos de Pensiones del año 1987, cuando se posibilitó la transformación voluntaria de fondos internos y otros sistemas de previsión del personal de las empresas en planes de pensiones.

46 Para elaborar esta gráfica, hemos partido de los datos contenidos en el Informe Estadístico de Instrumentos de Previsión Social Complementaria (2019 y avance 2020), publicado por el Ministerio de Asuntos Económicos y Transformación Digital. 
Pueden ser definidos como aquellos instrumentos de ahorro-previsión a largo plazo que promueve cualquier empresa o institución para sus empleados (partícipes), con la finalidad de complementar las pensiones públicas a percibir por el sistema de la Seguridad Social.

Según los datos del Ministerio de Asuntos Económicos y Transformación Digital, los planes de pensiones de empleo tenían, a fecha de 31 de diciembre de 2019, un patrimonio bajo gestión de 35.786 millones de euros y un número acumulado de cuentas de partícipes que superó la cifra de 2 millones ciento siete mil cuentas. La primera de estas dos cifras representa aproximadamente un 3\% del PIB anual de España, muy por debajo de otros países de la Unión Europea que cuentan con sistemas complementarios bastante más desarrollados.

El Gobierno español ha decidido revisar el modelo de previsión social complementaria en el ámbito empresarial y profesional, impulsando el ahorro a través de los planes de pensiones de empleo. Para ello, los Presupuestos Generales del Estado para el año 2021 incorporan estas dos propuestas:

- En primer término, un desplazamiento de las ventajas fiscales de los planes de pensiones individuales hacia los planes de pensiones de empleo procedentes de la negociación colectiva. De este modo, el límite de deducción por aportaciones a planes de pensiones individuales se rebaja de 8.000 a 2.000 euros anuales, mientras que el límite para los planes de empleo se eleva de 8.000 a 10.000 euros al año.

- En segundo lugar, la promoción de fondos de pensiones públicos de empleo de carácter abierto, que permitirán extender los planes de pensiones al conjunto de los trabajadores por medio de la negociación colectiva. Con estos nuevos fondos, se prevé aumentar la población cubierta por modelos complementarios (que, como se ha señalado, es mucho menor que la de otros países de nuestro entorno), atrayendo a rentas mediadas y bajas, así como a los jóvenes.

b. Seguros colectivos que instrumentan compromisos por pensiones de la empresa con trabajadores y beneficiarios (SC): pueden definirse como seguros colectivos de ahorro para dar cobertura a los compromisos por pensiones de la empresa con sus empleados. Se encuentran incluidos en este grupo los seguros concertados con las mutualidades de previsión social empresarial (MPSE).

A 31 de diciembre del año 2019, los seguros colectivos se concebían como el segundo instrumento más utilizado por las empresas para exteriorizar los compromisos por 
pensiones asumidos con sus trabajadores, contando con un patrimonio bajo gestión de 25.872 millones de euros y más de 8 millones y medio de cuentas, según datos facilitados por el Ministerio de Asuntos Económicos y Transformación Digital.

Su tratamiento fiscal es diferente al de los planes de pensiones de empleo y los planes de previsión social empresarial. Por este motivo, son compatibles en una misma empresa con estos otros dos instrumentos.

c. Planes de previsión social empresarial (PPSE): son otra de las soluciones de ahorro colectivo en la empresa más habituales. Se configuran como una modalidad de seguros colectivos de instrumentación de compromisos por pensiones y pueden cubrir las mismas contingencias que los planes de pensiones, ofreciendo un tipo de interés garantizado.

A finales del año 2019, los planes de pensiones de previsión social empresarial eran el tercer instrumento más usado para la exteriorización de compromisos por pensiones de las empresas con sus empleados. Tenían un patrimonio bajo gestión de 488 millones de euros y alrededor de 68 mil noventa y dos cuentas, según datos del Ministerio de Asuntos Económicos y Transformación Digital. Este importe de ahorro acumulado sigue siendo muy reducido en relación con el de los demás instrumentos de segundo pilar existentes (planes de pensiones de empleo y otros seguros colectivos).

Este instrumento se caracteriza por contar con los mismos incentivos fiscales que los ya mencionados planes de pensiones de empleo. De hecho, el régimen financiero y fiscal de las contingencias, aportaciones y prestaciones de los planes de previsión social empresarial se rige por la normativa referida a Planes y Fondos de Pensiones en todo lo que no se encuentre regulado específicamente por la Ley 35/2006, de 28 de noviembre, del Impuesto sobre la Renta de las Personas Físicas ${ }^{47}$ (en adelante, Ley del IRPF), y por el Real Decreto 439/2007, de 30 de marzo, por el que se aprueba el Reglamento del Impuesto sobre la Renta de las Personas Físicas ${ }^{48}$ (en lo sucesivo, Reglamento del IRPF).

\subsubsection{Pilar privado-individual}

\footnotetext{
${ }^{47} \mathrm{BOE} \mathrm{n}^{\circ} 285$, de 29 de noviembre de 2006.

${ }^{48} \mathrm{BOE}^{\circ}{ }^{\circ} 78$, de 31 de marzo de 2007.
} 
El llamado tercer pilar (vid. gráfica 5) se encuentra conformado por aquellos productos de previsión personal que la persona ahorradora decide contratar por iniciativa propia con una entidad financiera o compañía de seguros, generalmente sin la figura promotora de la empresa, institución o asociación. En virtud de ello, podemos señalar que no están ligados al desarrollo de la actividad profesional de la persona.

\section{Gráfica 5: Tercer pilar (Previsión individual)}

Privado, individual y voluntario

Principales magnitudes (2019)
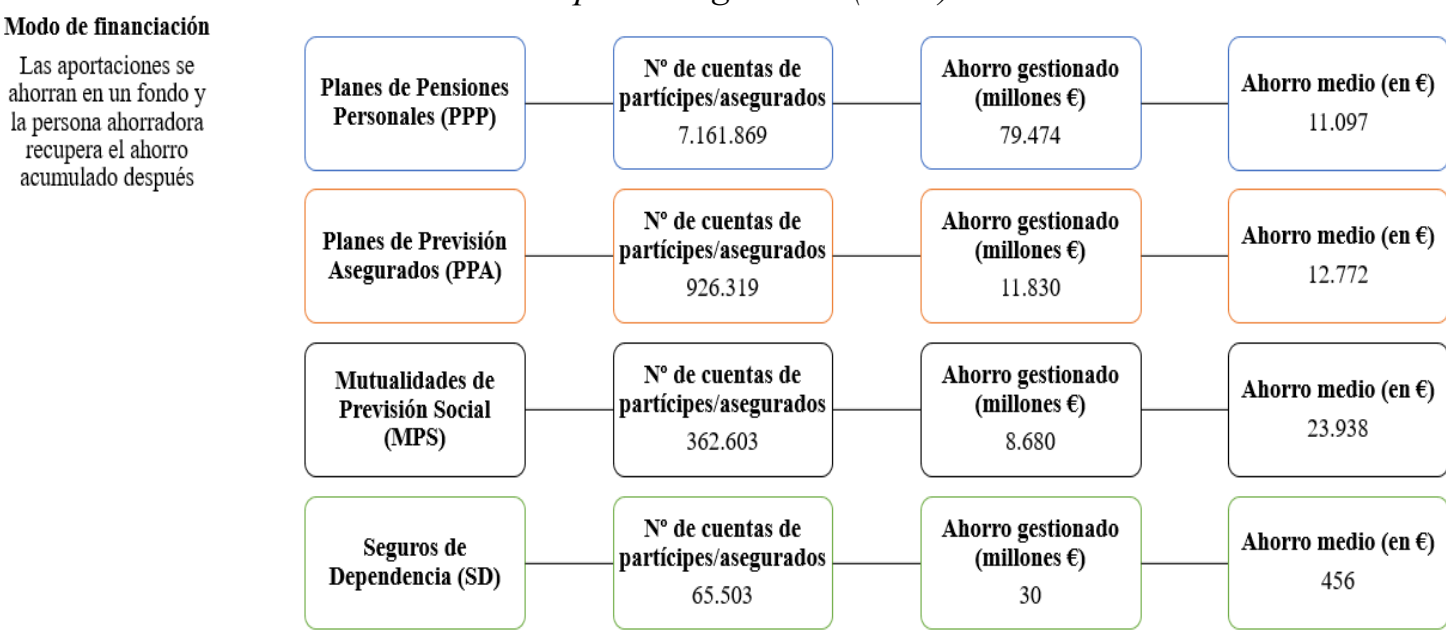

Gráfica de elaboración propia ${ }^{49}$.

Este pilar se caracteriza por ser privado, voluntario e individual. $\mathrm{Y}$ al igual que el pilar privado-colectivo, está basado en un sistema de capitalización, según el cual la persona partícipe se encarga de hacer una serie de aportaciones periódicas a lo largo de su vida activa que van generando su propia bolsa de ahorro (también conocida como "derechos consolidados", que podrán hacerse efectivos cuando acaezca alguna de las contingencias o supuestos que permiten el rescate). Las prestaciones a percibir van a depender tanto del valor de las aportaciones realizadas a lo largo del tiempo como del resultado de dicha capitalización.

Este pilar privado-individual conforma la fuente más relevante de la previsión social privada en España y se desarrolla a través de planes de pensiones individuales y asociados (los segundos son muy peculiares porque el promotor de los mismos es una asociación o sindicato y el partícipe es un asociado), que conjuntamente constituyen los planes de pensiones personales, planes de previsión asegurados, mutualidades de previsión social y seguros de dependencia. A finales de 2019, estos instrumentos (vid. gráfica 6) tenían un

\footnotetext{
${ }^{49}$ Para la elaboración de esta gráfica, hemos partido de los datos contenidos en el Informe Estadístico de Instrumentos de Previsión Social Complementaria (2019 y avance 2020), publicado por el propio Ministerio de Asuntos Económicos y Transformación Digital.
} 
patrimonio bajo gestión de 100.014 millones de euros y un número acumulado de cuentas de partícipes/asegurados de 8 millones quinientos dieciséis mil doscientos noventa y cuatro cuentas. A continuación, se examinan los referidos instrumentos.

\section{Gráfica 6: Ahorro gestionado}

Peso que en el año 2019 tenían los distintos instrumentos en el ahorro gestionado mediante el tercer pilar

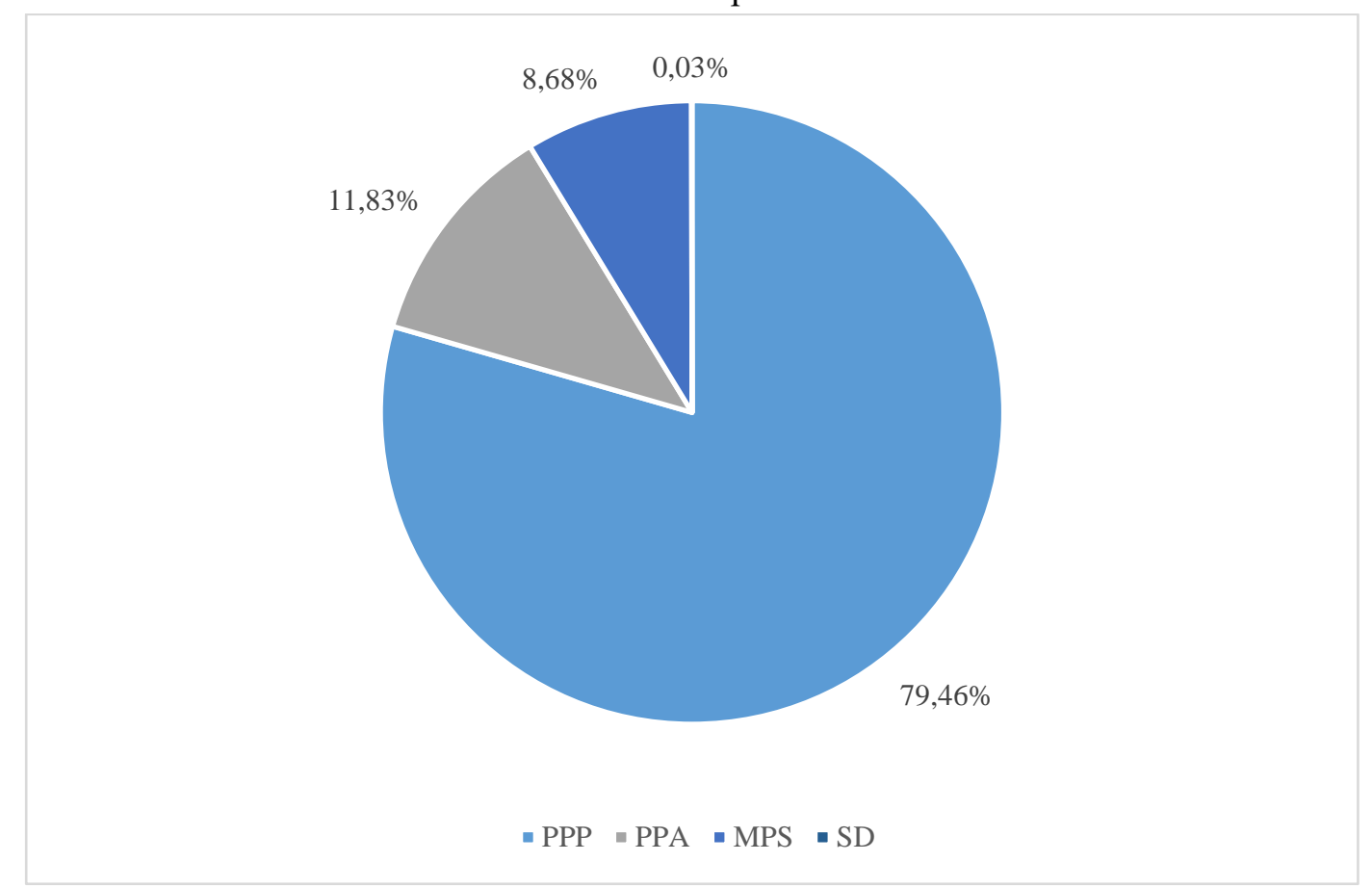

Gráfica de elaboración propia ${ }^{50}$.

a. Planes de pensiones personales (PPP): los planes de pensiones individuales y asociados son distintos productos de previsión privados enfocados principalmente en el ahorro para la jubilación. Considerados conjuntamente conforman lo que actualmente conocemos como planes de pensiones personales. Seguidamente, se definen estas dos modalidades:

- Sistema individual: en este primer caso, estamos hablando de los planes cuyo promotor es una o diferentes entidades de carácter financiero y cuyos partícipes son las personas físicas. Evidentemente, en este bloque se encuadran los planes de pensiones más conocidos en España, es decir, aquellos que se suscriben a iniciativa propia de los inversores mediante una entidad bancaria o cooperativa de crédito.

50 Para elaborar esta gráfica, hemos partido de los datos contenidos en el Informe Estadístico de Instrumentos de Previsión Social Complementaria (2019 y avance 2020), publicado por el Ministerio de Asuntos Económicos y Transformación Digital. 
- Sistema asociado: en este segundo caso, nos referimos a aquellos planes cuyo promotor es cualquier asociación, sindicato o colectivo, siendo los partícipes sus asociados, miembros o afiliados.

A 31 de diciembre del año 2019, los planes de pensiones personales eran concebidos como como el instrumento más utilizado para gestionar el ahorro voluntario incluido en el tercer pilar, contando con un patrimonio bajo gestión de 79.474 millones de euros y cerca de 7 millones doscientos mil cuentas de partícipes, según datos facilitados por el Ministerio de Asuntos Económicos y Transformación Digital.

En relación a las novedades fiscales, podemos señalar que la Ley 11/2020, de 30 de diciembre, de Presupuestos Generales del Estado para el año $2021^{51}$, que entró en vigor el 1 de enero del presente año, incorpora estos cambios que afectarán a todos los partícipes de planes de pensiones:

- Ha sido reducido a un máximo de 2.000 euros anuales el límite conjunto de reducciones por un mismo contribuyente (en el territorio común) por todas las aportaciones a los planes de pensiones personales. Hasta el 31 de diciembre del año 2020 este límite era el montante menor entre 8.000 euros anuales o el 30\% de los rendimientos netos de trabajo y de actividades económicas ${ }^{52}$.

- Es posible aumentar el nuevo límite de reducción de 2.000 euros en hasta 8.000 euros adicionales (hasta 10.000 euros en total; como ya se ha dicho anteriormente) por contribuciones del empleador a favor del empleado a instrumentos de previsión social empresarial (como planes de pensiones de empleo, planes de previsión social empresarial y mutualidades de previsión social). En este supuesto, también tendría que cumplir que como máximo sea el $30 \%$ de los rendimientos netos del trabajo. De este modo, si sumamos estos 8.000 euros, el límite máximo podrá alcanzar los 10.000 euros $^{53}$.

b. Planes de previsión asegurados (PPA): se definen en base al artículo 51.3 de la Ley del IRPF $^{54}$ como seguros individuales de vida especiales que permiten cubrir

\footnotetext{
${ }^{51} \mathrm{BOE} \mathrm{n}^{\circ} 341$, de 31 de diciembre de 2020.

52 Vid. BANKINTER, "Planes de Pensiones: cambios en la fiscalidad 2021 y alternativas para ahorrar para la jubilación”, Blog de Economía y Finanzas Bankinter, 2021 (disponible en: https://www.bankinter.com/blog/finanzas-personales/planes-pensiones-cambios-fiscalidad).

53 Ibídem.

${ }^{54}$ También se regulan en el artículo 49 del Reglamento del IRPF y en algunos aspectos en el Real Decreto $304 / 2004$, de 20 de febrero, por el que se aprueba el Reglamento de planes y fondos de pensiones (BOE ${ }^{\circ}$ 48 , de 25 de febrero de 2004).
} 
exactamente las mismas contingencias que los planes de pensiones (es decir, la jubilación, la incapacidad laboral, la dependencia, el fallecimiento, la enfermedad grave y el desempleo de larga duración), siendo la jubilación su principal cobertura. Ofrecen una rentabilidad mínima asegurada durante un determinado periodo de tiempo, pero, por lo general, la misma es peor que la de los planes de pensiones.

A finales del año 2019, los planes de pensiones de previsión social empresarial eran el segundo instrumento más usado para la gestión del ahorro voluntario incluido en el tercer pilar. Tenían un patrimonio bajo gestión de 11.830 millones de euros y de 926 mil trescientos diecinueve cuentas de asegurados, según datos del Ministerio de Asuntos Económicos y Transformación Digital.

Las aportaciones tienen idénticos límites que los planes de pensiones. De hecho, el límite máximo de aportación anual para un ahorrador aplica como límite conjunto. En el territorio común, el conjunto de las aportaciones anuales máximas realizadas a los sistemas de previsión social incluyendo, en su caso, las que hubiesen sido imputadas por los promotores, que puedan dar derecho a reducir la base imponible general no podrá exceder de 2.000 euros $^{55}$.

Será posible aumentar el referido límite de deducción de 2.000 euros en hasta 8.000 euros adicionales tan solo por contribuciones del empleador a favor del trabajador a instrumentos de previsión social empresarial (planes de pensiones de empleo, planes de previsión social empresarial y mutualidades de previsión social) ${ }^{56}$.

c. Mutualidades de previsión social (MPS): según el marco normativo aplicable (Real Decreto Legislativo 6/2004, de 29 de octubre, por el que se aprueba el texto refundido de la Ley de ordenación y supervisión de los seguros privados ${ }^{57}$ y Real Decreto 1430/2002, de 27 de diciembre, por el que se aprueba el Reglamento de mutualidades de previsión social ${ }^{58}$ ), pueden ser definidas como entidades aseguradoras privadas sin ánimo de lucro que ejercen una modalidad aseguradora de carácter voluntario y que cubren riesgos muy diversos.

Según los datos del Ministerio de Asuntos Económicos y Transformación Digital, las mutualidades de previsión social tenían, a fecha de 31 de diciembre de 2019, un

\footnotetext{
55 Vid. INSTITUTO BBVA DE PENSIONES, “Los planes de previsión asegurados (PPA)”, 2021 (disponible en: https://www.jubilaciondefuturo.es/es/blog/los-planes-de-prevision-asegurados-ppa-semejanzas-ydiferencias-con-los-planes-de-pensiones-pp.html).

${ }^{56}$ Ibídem.

${ }^{57}$ BOE $^{\circ} 267$, de 5 de noviembre de 2004.

${ }^{58} \mathrm{BOE} \mathrm{n}^{\mathrm{o}} 15$, de 17 de enero de 2003.
} 
patrimonio bajo gestión de 8.680 millones de euros y un número acumulado de cuentas de partícipes de 362 mil seiscientas tres cuentas.

d. Seguros de dependencia (SD): pueden ser definidos como contratos de seguro privados que cubren el riesgo de dependencia severa o de gran dependencia conforme a la definición legal de dichas contingencias (vid. el artículo $26^{59}$ de la Ley 39/2006, de 14 de diciembre, de Promoción de la Autonomía Personal y Atención a las personas en situación de dependencia ${ }^{60}$ ), pudiendo generar también prestaciones por fallecimiento. Dichos contratos de seguro tendrán obligatoriamente que ofrecer una garantía de interés y utilizar técnicas actuariales.

A 31 de diciembre de 2019, tenían un patrimonio bajo gestión de tan solo 30 millones de euros y algo más de 65 mil quinientas cuentas de asegurados, de acuerdo con los datos ofrecidos por el Ministerio de Asuntos Económicos y Transformación Digital.

El régimen fiscal de las aportaciones es similar al de los planes de pensiones (vid. artículo 51.5 de la mencionada Ley del IRPF). En los seguros privados que cubren exclusivamente el riesgo de dependencia severa o de gran dependencia, el conjunto de las reducciones practicadas por todas las personas que satisfagan primas a favor de un mismo contribuyente, incluidas las del propio contribuyente, no podrán exceder de 2.000 euros anuales (con anterioridad el límite era de 8.000 euros anuales).

\section{Para concluir: ¿hacia dónde vamos?}

En virtud de lo expuesto en los diferentes epígrafes de este trabajo, se puede concluir que el primer pilar analizado, es decir, el sistema público de prestaciones de la Seguridad Social constituye en la actualidad el núcleo principal de nuestro modelo de previsión social, ya que en torno al mismo (concretamente, en torno al subsistema contributivo) gira la política de pensiones española.

\footnotetext{
${ }^{59}$ El artículo 26 se refiere a los grados de dependencia y establece que: "la situación de dependencia se clasificará en los siguientes grados: (...) b) Grado II. Dependencia severa: cuando la persona necesita ayuda para realizar varias actividades básicas de la vida diaria dos o tres veces al día, pero no quiere el apoyo permanente de un cuidador o tiene necesidades de apoyo extenso para su autonomía personal. c) Grado III. Gran dependencia: cuando la persona necesita ayuda para realizar varias actividades básicas de la vida diaria varias veces al día y, por su pérdida total de autonomía física, mental, intelectual o sensorial, necesita el apoyo indispensable y continuo de otra persona o tiene necesidades de apoyo generalizado para su autonomía personal. (...)". ${ }^{60} \mathrm{BOE} \mathrm{n}^{\mathrm{o}} 299$, de 15 de diciembre de 2006.
} 
En nuestra opinión, el sistema público de pensiones mantendrá un peso mayoritario en el futuro y seguirá cumpliendo la finalidad que ha tenido siempre, que no es otra que garantizar que no se encuentre ninguna persona en nuestro país en situación de necesidad. En este sentido, creemos que las pensiones públicas tendrían que seguir conformando el principal programa redistributivo del modelo español de previsión social y, de este modo, entendemos que deberían seguir contribuyendo firmemente a reducir la desigualdad y a prevenir el riesgo de pobreza de muchas personas como las de mayor edad.

Ahora bien, aunque es muy probable que el sistema público de pensiones español siga manteniendo un peso muy importante en el futuro, nos preocupa enormemente que las sucesivas reformas que se lleven a cabo en nuestro país lo sigan recortando -como hasta ahora ha ocurrido-, desplazando aún más las fórmulas de reparto hacia las privadas o de capitalización (como las que hemos estudiado en este trabajo) que cuentan con numerosos inconvenientes.

\section{Bibliografía}

Aguilar Segado, Carlos David, "Situación actual de las pensiones en España: perspectiva económica-financiera", Revista de Estudios Jurídico Laborales y de Seguridad Social, nº 2, 2021.

ÁlVAREZ CORTÉS, Juan Carlos., "La pensión de jubilación no contributiva: su futuro papel de malla de seguridad social ante el repliegue contributivo", en AA.VV. (ÁlvAREz CORTÉS, Juan Carlos, Dir.), Trabajadores maduros y Seguridad Social, Thomson Reuters Aranzadi, Cizur Menor, 2018.

BANKINTER, "Planes de Pensiones: cambios en la fiscalidad 2021 y alternativas para ahorrar para la jubilación”, Blog de Economía y Finanzas Bankinter, 2021 (disponible en: https://www.bankinter.com/blog/finanzas-personales/planes-pensiones-cambiosfiscalidad).

BERRAQUERO ESCRIBANO, Iván, "La supresión de las clases pasivas. Implicaciones a largo plazo para las Comunidades Autónomas", ASOCEX (disponible en: https://asocex.es/la-supresion-de-las-clases-pasivas-implicaciones-a-largo-plazo-paralas-comunidades-autonomas).

DuPEYroux, Jean Jacques, Évolution et tendances des systèmes de Sécurité Sociale des pays membres des Communautés Européennes et de la Grande-Bretagne, Services des publications des Communautés Européennes, Luxembourg, 1966 (disponible en: http://aei.pitt.edu/36107/1/A2119.pdf). 
GARCÍA PADRÓN, Yaiza y GARCÍA BOZA, Juan, “Análisis legislativo de los planes y fondos de pensiones en España. Instauración y desarrollo", Ciencia y Sociedad, vol. XXXI, n 2 , 2006.

Gillion, Colin, Turner, John, Bailey, Clive y Latulippe, Denis, Social Security pensions: development and reform, International Labour Organization, Geneva, 2000.

GutiérRez BengoecheA, Miguel, "El sistema de reparto de la Seguridad Social versus envejecimiento poblacional", Revista de Estudios Jurídico Laborales y de Seguridad Social, $\mathrm{n}^{\mathrm{o}}$ 1, 2020.

Gutiérrez BengoecheA, Miguel, "Fiscalidad de los planes de pensiones privados", Revista de Derecho de la Seguridad Social, n² 26, 2021.

Hernández Solís, Montserrat, Topa CAntisano, Gabriela y Herrador AlCAide, Teresa Carmen, "Análisis de la actitud financiera personal para la jubilación: un sondeo en España”, Economía industrial, no 413, 2019.

Instituto BBVA DE Pensiones, "Los planes de previsión asegurados (PPA)", 2021 (disponible en: https://www.jubilaciondefuturo.es/es/blog/los-planes-de-previsionasegurados-ppa-semejanzas-y-diferencias-con-los-planes-de-pensiones-pp.html).

Instituto BBVA De Pensiones, "Los tres pilares de la previsión social: del ámbito público al privado", 2019 (disponible en: https://www.jubilaciondefuturo.es/es/blog/lostres-pilares-de-la-prevision-social-del-ambito-publico-al-privado.html).

Maldonado Molina, Juan Antonio, "Las pensiones no contributivas", en AA.VV., Tratado de Derecho de la Seguridad Social, tomo II, Laborum, Murcia, 2017.

Monereo PÉrez, José Luis, "La garantía de las pensiones: desafíos para la sostenibilidad económica y social”, Revista de Estudios Jurídico Laborales y de Seguridad Social, no 3 , 2021.

Monereo PÉrez, José Luis et al., Manual de Seguridad Social, Tecnos, Madrid, 2020.

Monereo Pérez, José Luis y Fernández Bernat, Juan Antonio, "Planes y fondos de pensiones", en AA.VV., Tratado de Derecho de la Seguridad Social, tomo II, Laborum, Murcia, 2017. 
Monereo Pérez, José Luis, OjedA Avilés, Antonio y Gutiérrez BengoecheA, Miguel, Reforma de las pensiones públicas y planes privados de pensiones, Laborum, Murcia, 2021.

Moreno Gené, Josep y Romero Burillo, Ana María, "Los planes y fondos de pensiones ante las nuevas formas de organización de la empresa", Revista de trabajo y seguridad social CEF, $\mathrm{n}^{\mathrm{o}} 245-246,2003$.

MORENO ROMERO, Francisca, "Requisitos generales de acceso a la protección y sus vicisitudes: hacia un necesario replanteamiento", en AA.VV. (VILA TIERNO, Francisco, y Gutiérrez Bengoechea, Miguel, (Directores); Gómez SAlado, Miguel Ángel, Coordinador), La incidencia de los diferentes factores endógenos y exógenos sobre sostenibilidad y suficiencia en el sistema de pensiones, Comares, Granada, 2020.

NATALI, David, "La reforma de las pensiones en la Unión Europea: Cambios en las políticas nacionales y coordinación de la Unión Europea”, en AA.VV. (FrADES PERNAS, Jaime, Coordinador), El sistema público de pensiones de jubilación. Desafios y respuestas, Fundación Largo Caballero, Madrid, 2011.

Pieschacón Velasco, Camilo, La Ley de Planes y Fondos de Pensiones: 20 Años después, Fundación Inverco, Madrid, 2007.

Pieschacón Velasco, Camilo, Sistemas de pensiones: experiencia española e internacional, tomo II, Fundación Inverco, Madrid, 2005.

REDACCIÓN DE EKONOMIAZ, "Guía de la previsión social complementaria”, Revista vasca de economía (Ekonomiaz), nº 85, 2014.

Rodríguez CABrero, Gregorio, "La reforma del sistema público de pensiones en España”, Documentos de trabajo (CSIC. Unidad de Políticas Comparadas), nº 13, 2002.

Rodríguez CABrero, Gregorio, "La reforma permanente del sistema público de pensiones", en AA.VV., Actores sociales y reformas del bienestar, CSIC, Madrid, 2005. RoDRÍGUEZ CANFRANC, Miguel, "¿Qué son los sistemas de pensiones de aportación definida?", $B B V A$, agosto de 2020 (disponible en: https://www.bbva.com/es/que-son-lossistemas-de-pensiones-de-aportacion-definida/).

RUIZ SANTAMARÍA, José Luis, "Las pensiones en el marco del actual Convenio Bilateral de Seguridad Social suscrito entre España y Colombia”, e-Revista internacional de la protección social, vol. $3, \mathrm{n}^{\mathrm{o}} 2$. 
SÁnchez-RodAs NAVARro, Cristina, "La cuadratura del círculo: sostenibilidad del sistema de pensiones y desempleo juvenil”, Revista Galega de Dereito Social, nº 2, 2016.

Sitjar DE TOGORES CALvo, Ana Isabel., "Planes de pensiones: cuestiones transfronterizas. Una aproximación a la normativa y jurisprudencia de la Unión Europea”, Cuadernos de información, vol. 7, 2009.

TORTUERO PlazA, José Luis, "Los retos históricos del sistema de pensiones proyectados en tiempos de crisis económico financiera", Areas: revista internacional de ciencias sociales, $\mathrm{n}^{\mathrm{o}} 32,2013$.

VILA TIERNO, Francisco, "El difícil equilibrio entre la sostenibilidad y la suficiencia. Una visión general de la situación general del sistema de pensiones pensando en el futuro", en AA.VV. (Vila Tierno, Francisco, y Gutiérrez Bengoechea, Miguel, (Directores); Gómez SAlado, Miguel Ángel, Coordinador), La incidencia de los diferentes factores endógenos y exógenos sobre sostenibilidad y suficiencia en el sistema de pensiones, Comares, Granada, 2020.

Vila Tierno, Francisco y Moreno Romero, Francisca, "Equilibrio entre suficiencia y sostenibilidad: pensiones mínimas, revalorización automática y factor de sostenibilidad”, en AA.VV. (ÁlvAREz CORTÉs, Juan Carlos, Director), Trabajadores maduros y Seguridad Social, Thomson Reuters Aranzadi, Cizur Menor, 2018. 